\title{
Interannual to Decadal Predictability in a Coupled Ocean-Atmosphere General Circulation Model
}

\author{
A. GrötZner, M. Latif, And A. Timmermann \\ Max-Planck-Institut für Meteorologie, Hamburg, Germany \\ R. Voss \\ Deutsches Klimarechenzentrum, Hamburg, Germany
}

(Manuscript received 10 July 1998, in final form 1 December 1998)

\begin{abstract}
The predictability of the coupled ocean-atmosphere climate system on interannual to decadal timescales has been studied by means of ensemble forecast experiments with a global coupled ocean-atmosphere general circulation model. Over most parts of the globe the model's predictability can be sufficiently explained by damped persistence as expected from the stochastic climate model concept with damping times of considerably less than a year. Nevertheless, the tropical Pacific and the North Atlantic Ocean exhibit oscillatory coupled ocean-atmosphere modes, which lead to longer predictability timescales. While the tropical mode shares many similarities with the observed ENSO phenomenon, the coupled mode within the North Atlantic region exhibits a typical period of about $30 \mathrm{yr}$ and relies on an interaction of the oceanic thermohaline circulation and the atmospheric North Atlantic oscillation. The model's ENSO-like oscillation is predictable up to one-third to onehalf $(2-3$ yr) of the oscillation period both in the ocean and the atmosphere. The North Atlantic yields considerably longer predictability timescales (of the order of a decade) only for quantities describing the model's thermohaline circulation. For surface quantities and atmospheric variables only marginal predictability (of the order of a year) was obtained. The predictability of the coupled signal at the surface is destroyed by the large amount of internally generated (weather) noise. This is illustrated by means of a simple conceptual model for coupled oceanatmosphere variability and predictability.
\end{abstract}

\section{Introduction}

The earth's climate is characterized by considerable variability on timescales ranging from seasons to decades. To predict these fluctuations is a major task of current climate research. The internal variability of the atmosphere by itself, as it is addressed by deterministic weather prediction, is predictable only over a period of one or two weeks in advance due to the nonlinear and instability properties of the atmospheric flow. Nevertheless, climate fluctuations might be potentially predictable over much longer periods of months or even years due to coupling to slower components of the climate system. In the context of seasonal to decadal timescales the world oceans are the most hopeful candidates to represent such a long-term memory of the climate system.

A basic concept to understand climate variability and

Corresponding author address: Dr. A. Grötzner, Max-Planck-Institut für Meteorologie, Bundesstrasse 55, D-20146 Hamburg, Germany.

E-mail: groetzner@dkrz.de predictability is given by Hasselmann's (1976) stochastic climate model approach. In its well-known application to air-sea interaction, atmospheric "noise" (the high-frequency weather fluctuations) is thought to be integrated by slowly responding components of the ocean, for example, by the oceanic mixed layer. This leads to low-frequency fluctuations in the ocean greater than expected from the random forcing. Internal damping processes limit these excursions from growing to infinity. This mechanism bears fundamental similarities to the classical random walk model for Brownian motion and can mathematically be expressed by a first-order Markov process. The redness of observed sea surface temperature (SST) spectra, for example, can sufficiently be explained within the stochastic climate model framework. Due to the memory of the system it is possible to derive useful forecasts of oceanic quantities (such as SST) for times much longer than the predictability limit of atmospheric weather, that is, about two weeks. Typical decorrelation timescales for such damped persistence forecasts are of the order of several months in the case of mixed-layer processes. The stochastic climate model concept can be extended to other even slower processes, as it has been recently done by Frankignoul 
et al. (1997), who incorporated the wind-driven ocean gyres, which extends the applicability of the stochastic climate model to decadal timescales.

The basic stochastic climate model concept is able to explain a large portion of observed climate fluctuations and can be viewed as a kind of a "null-hypothesis" for the generation of natural climate variability. However, other types of variability are still possible. Oscillations relying on delayed feedbacks may exist. In contrast to a first-order stochastic process, such oscillations are related to specific timescales. Damped eigenmodes of the ocean circulation can be exited by the atmospheric noise forcing, as suggested, for instance, by the modeling studies by Mikolajewicz and Maier-Reimer (1990), Delworth et al. (1993), and Griffies and Tziperman (1995). Besides such a one-sided influence of the atmosphere on the ocean, "two-way" interactions, where both ocean and atmosphere influence each other, can also be important for the generation of climate variability. The most prominent interannual climate fluctuation, the El Niño-Southern Oscillation (ENSO) phenomenon, relies on such a coupled feedback loop including both the tropical ocean and atmosphere. Two-way interactions are also proposed to cause decadal climate variability over the North Pacific (Latif and Barnett 1994; Gu and Philander 1997), in the tropical Atlantic (Chang et al. 1997), and the North Atlantic Ocean (e.g., Grötzner et al. 1998; Timmermann et al. 1998). Both in the case of one-way and two-way interactions, the memory of the coupled system resides in the ocean. Since many damping processes like diffusion or nonlinearities are acting in the climate system, these oscillations can usually not be expected to be self-sustained. The forcing necessary to overcome the inherent damping of the system is provided by the internal noise of the coupled ocean-atmosphere system, especially by the random weather fluctuations.

Depending on the strength of the damping such stochastically forced damped oscillators can be much more predictable than simple first-order processes. For instance, it is now possible by means of statistical and physically based prediction models to predict the onset of ENSO 6-12 months in advance [an overview is given in Latif et al. (1998)]. Since ENSO represents such a strong signal, its influence is not limited to the tropical Pacific, and it has also significant impacts over other parts of the globe, for example, the North Pacific and North America. Therefore, forecasts of ENSO are also relevant in those areas (Barnett and Preisendorfer 1987; Barnston 1994).

However, the prediction of climate fluctuations in the extratropics is a much more complicated problem than in the Tropics. While a large amount of the variability of the tropical atmosphere can be explained by linear dynamics, the circulation of the extratropical atmosphere is characterized by nonlinearities and internal instabilities. These processes cause a large amount of unpredictable internal variability or noise. The moti- vation for extratropical climate predictions relies on the expectation, that some of the long-term signals, which might either originate from the Tropics as in the case of ENSO or from a coupling to the underlying ocean, may be large enough to be significant against the background noise. Therefore, the task of making climate predictions has to be understood as a problem of separating some predictable signal from unpredictable noise.

Many papers based on both modeling and observations have addressed the predictability of the tropical ENSO phenomenon (Latif et al. 1998). A first modeling attempt dealing with the predictability of extratropical climate fluctuations on interannual to decadal timescales has been performed by Griffies and Bryan (1997a,b). The interdecadal variability of the central North Atlantic Ocean, as it is simulated by the coupled ocean-atmosphere model of the Geophysical Fluid Dynamics Laboratory (GFDL), is characterized by an oscillation of the thermohaline meridional overturning circulation with a timescale of about $50 \mathrm{yr}$ (Delworth et al. 1993). Delworth et al. (1997) describe coherent variability in the Greenland Sea related to intensity fluctuations in the East Greenland current and an impact on temperature and wind fields in the overlying atmosphere. They also indicate that the ocean circulation anomalies might be triggered by the atmosphere. By means of ensemble experiments starting from perturbed initial conditions, Griffies and Bryan (1997a,b) have studied the North Atlantic climate predictability related to these climate fluctuations. Their results yield predictability timescales up to $20 \mathrm{yr}$ for quantities describing the large-scale oceanic circulation. Griffies and Bryan (1997a,b) interpret the physical mechanism leading to their simulated North Atlantic climate fluctuations as eigenoscillations of the oceanic circulation itself. The atmosphere is not considered as an active element of the feedback loop. The atmosphere only provides stochastic forcing to overcome the damping of the system. Therefore, it is plausible that Griffies and Bryan (1997a,b) did not address the issue of atmospheric predictability at decadal timescales.

The interannual to decadal climate variability has been studied also in a millennium integration with the coupled ocean-atmosphere general circulation model ECHAM3-LSG, which has been performed at the Deutsches Klimarechenzentrum (Voss et al. 1998). Although the model has a rather coarse resolution, it exhibits interannual variability within the tropical Pacific similar to the ENSO phenomenon (Timmermann et al. 1999, manuscript submitted to J. Climate; hereafter TLGV99). Within the North Atlantic region the model reveals also oscillations of the coupled ocean-atmosphere system. Timmermann et al. (1998) and Timmermann (1999) describe a wind-driven mode with 15yr period similar to that described by Grötzner et al. (1998) and a thermohaline driven coupled mode with 35-yr period. The North Pacific Ocean reveals also rath- 
er regular interdecadal decadal variability. However, these fluctuations cannot be associated with the coupled phenomenon described by Latif and Barnett (1994) and Latif and Barnett (1996), but are more likely due to a remote response to the interdecadal variability in the North Atlantic.

The fact that these climate modes involve the atmosphere as an essential component gives rise for the hope that the extratropical atmosphere might be at least to some extent predictable on decadal timescales. Similar to Griffies and Bryan (1997a,b), we have performed ensemble forecast experiments starting from perturbed initial conditions of a control run to assess the limits of the models oceanic and atmospheric predictability. In section 2 we describe the coupled model and the setup used for the ensemble experiments. Section 3 gives a brief overview of the model's North Atlantic variability and describes the results concerning the predictability of these phenomena. In section 4 we extend our analyses to the whole globe. A summary and discussion conclude the paper in section 5 .

\section{The coupled model and experimental setup}

Our experiments are based on a millennium integration with the global coupled ocean-atmosphere model ECHAM3-LSG. The model consists of the atmospheric general circulation model ECHAM3, the Hamburg version of the European Center operational weather forecast model with physical parameterizations modified for climate modeling purposes (Roeckner et al. 1992). ECHAM3 is a global low-order spectral model that we used at a triangular truncation at wavenumber 21 (T21). This corresponds to a horizontal resolution of about $5.6^{\circ}$. The model was run with 19 levels in the vertical.

The ocean model is the Large Scale Geostrophic model (LSG; Maier-Reimer et al. 1993). The LSG model is based on primitive equations. The nonlinear advection of momentum, however, is neglected. This assumption provides an appropriate representation of the slow ocean modes only, so that the model becomes very efficient. The model has 11 levels in the vertical and is integrated using a fully implicit numerical scheme on a horizontal E-grid with a resolution of about $4^{\circ}$. A thermodynamic sea-ice model is included. To avoid climate drift in the coupled mode, the flux correction technique (Sausen et al. 1988) is applied, which is equivalent to coupling both subsystems by their individual flux anomalies relative to their equilibrium states. The ECHAM3-LSG model has been used in a number of climate change and response experiments (e.g., Hasselmann et al. 1995; Cubasch et al. 1997; Schiller et al. 1997). The first $500 \mathrm{yr}$ of the long-term integration are described in detail in Voss et al. (1998). We shall discuss certain aspects of the models interannual and interdecadal variability in connection with the results of the predictability experiments within the following sections.

The prediction of dynamical systems is limited to certain time intervals due to system inherent uncertainties. Beyond that time limit the system can be viewed to be independent from its initial conditions. Slight changes in the initial conditions lead to different time behavior of the system. The initial differences grow and disturb the memory provided by the imposed initial conditions. A widely used method to explore such predictability limits with numerical models is the classical ensemble forecast approach first proposed by Lorenz (1963). An ensemble of realizations is started from slightly perturbed initial conditions. The individual system trajectories will diverge up to a certain time, then their probability density distribution becomes indistinguishable from that of climatology. As long as the variance within the ensemble remains small compared to the climatological variance, the system can be viewed to be predictable.

Many studies have addressed the ENSO predictability limits that originate from imperfectly known initial states due to incomplete observations and compared model forecasts with actual observations. Here we consider a more principal issue. We try to investigate the theoretical predictability limits that arise from uncertainties within our coupled ocean-atmosphere model itself. Due to instabilities and nonlinearities, the atmospheric flow, especially in the extratropics, is characterized by a rather irregular behavior. Thus, the predictability of atmospheric weather fluctuations is limited to 1-2 weeks, and for longer timescales the atmospheric weather can be viewed as random stochastic forcing acting on the ocean. The oceanic circulation is much less affected by such irregularities, although the internal oceanic variability is in general underestimated by current large-scale ocean circulation models. We view the atmospheric flow as the main agent in introducing uncertainties into the coupled system. Therefore, we have perturbed the atmospheric initial conditions only within the ensembles. To obtain these initial conditions the atmosphere-only component of the model has been forced by the fixed initial SST distribution and integrated over a limited time period of a few months. The weather fluctuations within this time interval yield different realizations of the state of atmosphere compatible with the initial SST field. Samples of these atmospheric states, separated by 5 days to avoid atmospheric autocorrelation, have been used as initial conditions for the predictability experiments. The oceanic initial conditions were chosen to be identical for all members of an individual ensemble. Hence, our experimental setup yields an upper limit of interannual to decadal predictability, since the assumption of a perfect knowledge of the oceanic initial conditions will be never fulfilled in a real forecast situation.

We have chosen four states from the first $700 \mathrm{yr}$ of the control integration as starting points for our ensemble experiments, from which the coupled model was restarted. Each ensemble consists of 11 individual realizations that have been integrated over $30 \mathrm{yr}$. One 
ensemble, however, consists of members of 15-yr length only due to limitations in computer resources. We shall show results from all four forecasts ensembles. Results derived from time series of area-averaged quantities and from patterns derived from EOF analyses, which describe the leading modes of the model's long-term climatic variability will be shown. The predictability will be investigated by comparing trajectories of individual ensemble members, the temporal evolution of the ensemble mean, and the normalized variance within each of the four ensembles. The variances are normalized using the respective variance computed from the first $700 \mathrm{yr}$ of the control integration. When the normalized ensemble variance approaches unity, the variations between individual ensemble members become indistinguishable from the climatological variability of the control run. This defines a theoretical predictability limit. It should be noted, however, that the "useful" predictability limit may be much shorter than the theoretical limit. Since we accept the stochastic climate model as a null hypothesis for natural climate variability and predictability, we shall always compare our model results to those that are obtained by fitting equivalent first-order autoregressive processes.

\section{Prediction of Northern Atlantic climate fluctuations}

Stochastic considerations yield three basic cases of predictability of the ocean-atmosphere system. In the case of the pure stochastic climate model the predictability limit of oceanic quantities is given by their decorrelation timescale (damped persistence), which ranges typically from several months at the surface of the ocean to many years within the oceans interior. The predictability limit of the atmosphere is given by that of individual weather phenomena (about two weeks), since no feedback from the ocean to the atmosphere is considered. If a stochastically forced "ocean-only" oscillator is considered, enhanced predictability relative to damped persistence is found around the resonance frequency. Predictability limits of atmospheric variables are the same as in the first case. However, both cases can be extended by considering that the atmosphere might react passively to the oceanic anomalies. This might introduce some climate predictability in the atmosphere beyond the limits given by atmospheric weather. If the atmosphere both reacts to oceanic anomalies and passes the signal back to the ocean, a coupled ocean-atmosphere oscillator can be formed. In this case we find the quasi-periodic signal both in the ocean and the atmosphere. Thus, it can be expected that in this case both the ocean and the atmosphere might reveal enhanced predictability relative to damped persistence at the resonance frequency. It depends critically on the degree of the damping, whether the coupled modes can significantly affect the predictability of the atmosphere on climatic timescales. Since we found coupled ocean- atmosphere modes of the North Atlantic climate system with decadal timescales in the extended-range integration with the ECHAM3-LSG coupled model, we have tried to investigate their predictability.

The long-term memory of the ocean circulation in the North Atlantic can be conceptually separated into a "wind-driven" and a "thermohaline" part. The former is forced by the surface wind stress and associated with horizontal circulations in the upper ocean (subtropical and subpolar gyres), while the latter is associated with a meridional (north-south) circulation and deep convection in the northern North Atlantic. An analysis of the first $700 \mathrm{yr}$ of the integration with the ECHAM3LSG coupled model reveals variations in both types of circulation systems (Timmermann 1999; Timmermann et al. 1998). The adjustment times of the gyre circulations are generally shorter than those of the thermohaline circulation (THC). Variations associated with the gyres have typical timescales of about 10-20 yr, while those linked to the THC have typical timescales of several decades. Interestingly, both types of variability seem to coexist in the coupled model simulation, and there are some indications from observations that this might be the case in the real world, too (e.g., Deser and Blackmon 1993; Kushnir 1994).

Spectra of simulated Northern Atlantic climate variables are in general consistent with the stochastic climate model hypothesis. Atmospheric spectra are almost white while oceanic spectra show a red noise behavior. Two spectral peaks, however, have been discovered. Timmermann (1999) and Timmermann et al. (1998) have related these peaks to two coupled ocean-atmosphere oscillations with $15 \mathrm{yr}$ and 30-40-yr timescales. The 15-yr peak is related to a coupled feedback loop that basically involves the wind-driven gyre circulation, unstable air-sea interactions, and the atmospheric North Atlantic Oscillation (NAO). This mode shares many similarities with the phenomena described by Latif and Barnett (1996) and Grötzner et al. (1998). However, this eigenmode of the coupled system is only marginally significant in the ECHAM3-LSG simulation. Hence, it is reasonable to assume that this decadal mode is strongly damped and that the bulk of the decadal variability in the range 10-20 yr can sufficiently be explained within the stochastic climate model scenario. We have analyzed this mode with respect to possible impacts on North Atlantic climate predictability. However, it turned out that the damping of this mode is too strong to yield any impacts on the model's predictability. For this reason we shall not focus on this mode in the following.

The peak at the longer timescale is much more pronounced and deviates significantly from the stochastic climate model null hypothesis. It is related to a coupled air-sea mode (Timmermann et al. 1998), and the memory of the coupled system resides in the model's THC. Figures 1a and $1 \mathrm{~b}$ show the first EOF of the zonally averaged streamfunction in the North Atlantic and the spectrum of the corresponding principal component 


\section{Meridional Streamfunction}

a)

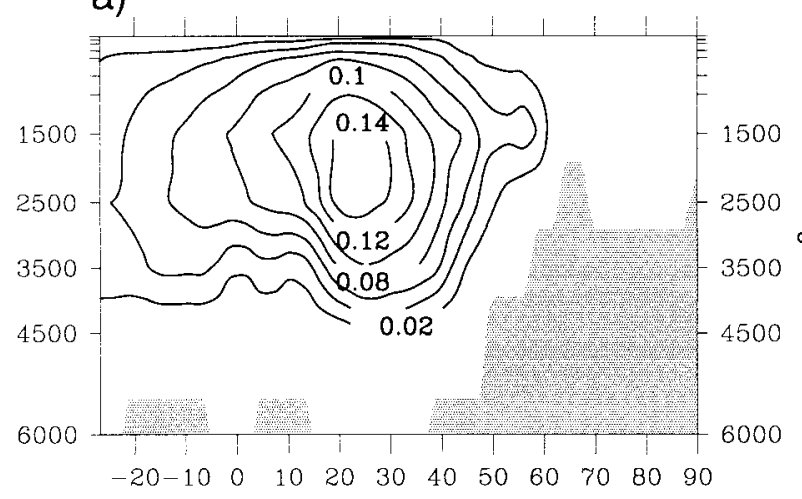

b) Spectrum of PC1

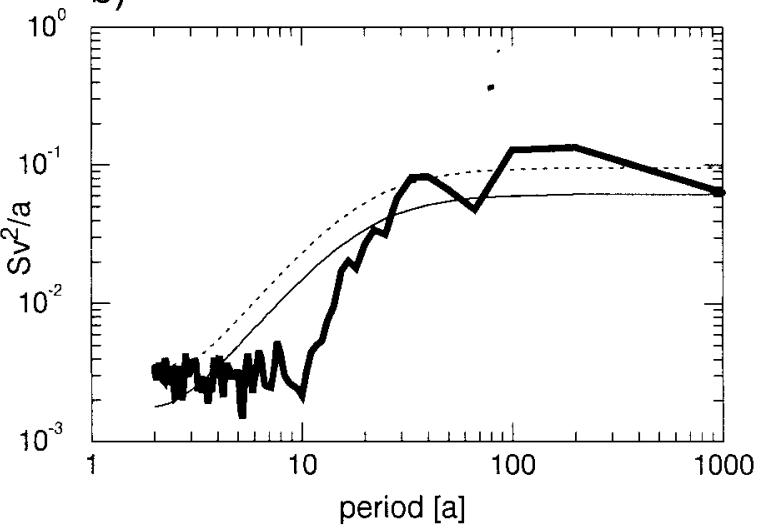

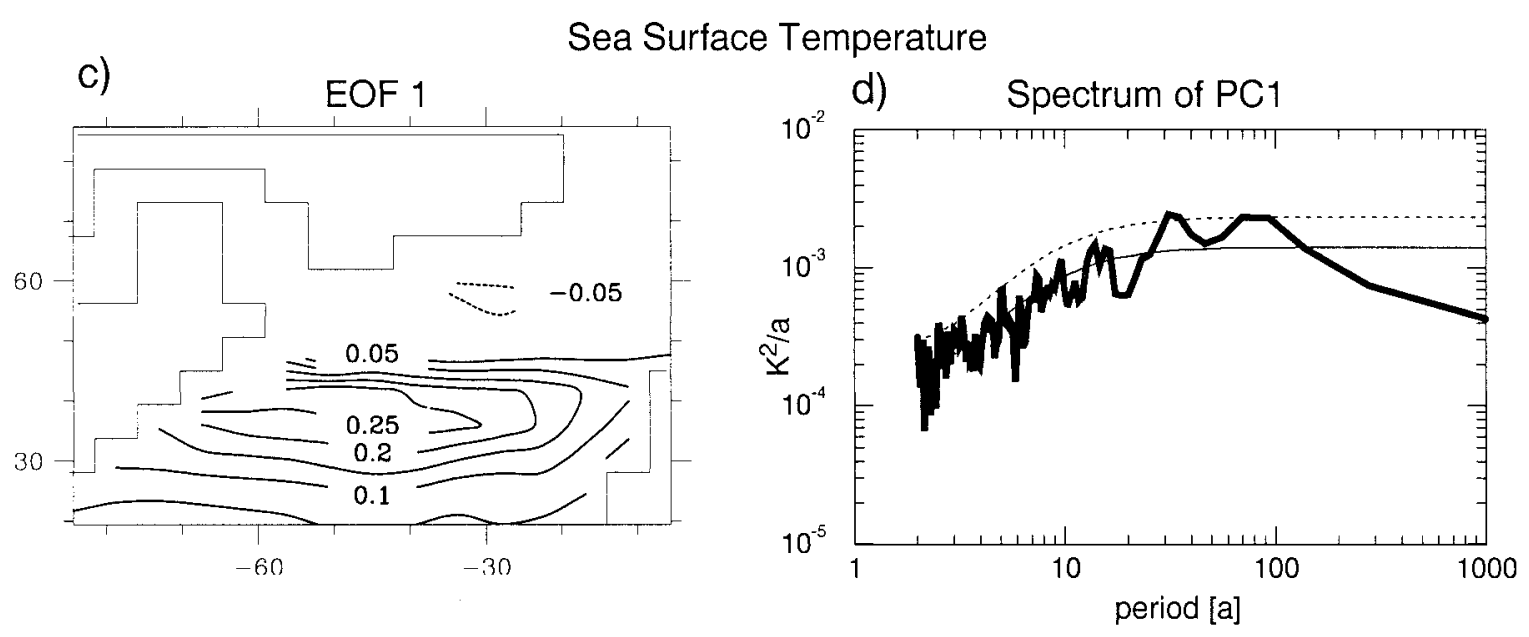

$500 \mathrm{hPa}$ Geopotential Height

e)

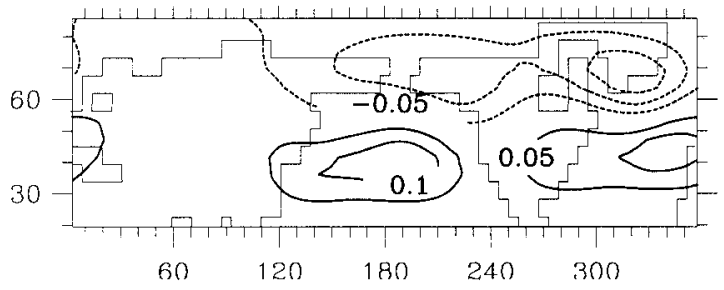

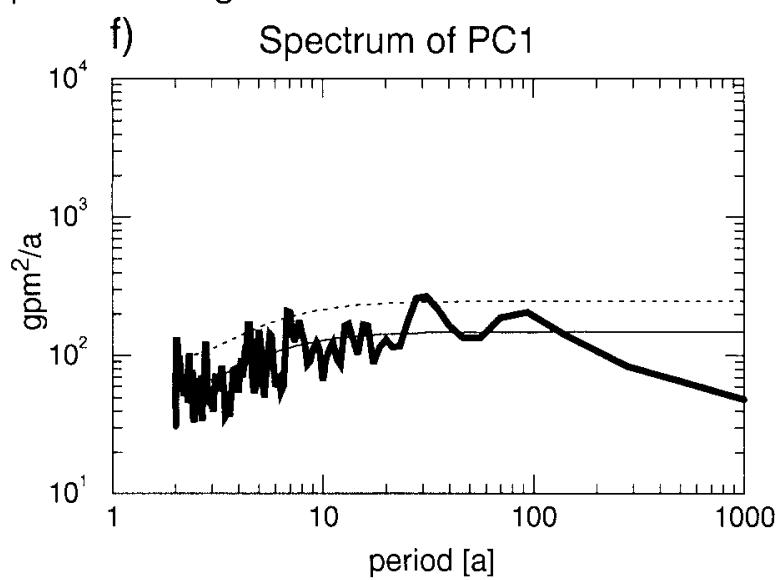

FIG. 1. (a) First EOF of zonally averaged North Atlantic streamfunction and (b) spectrum of accompanying principal component time series, (c) first EOF of SST and (d) accompanying spectrum, and (e) first EOF of Northern Hemispheric 500-hPa geopotential height and (f) accompanying spectrum as simulated by the ECHAM3-LSG model. The standard deviations of the corresponding principal components are $6.71 \mathrm{~Sv}\left(\mathrm{~Sv} \equiv 10^{6} \mathrm{~m}^{3} \mathrm{~s}^{-1}\right), 1.67 \mathrm{~K}$, and $114 \mathrm{gpm}$, respectively. 
based on annual-mean values. The variability of the meridional circulation is dominated (explained variance is $65 \%$ ) by intensity variations of the overturning cell. The spectrum clearly shows a large amount of variance at the 30-yr timescale. Timmermann et al. (1998) gave a slightly longer timescale for this oscillation, but their analysis was based on $700 \mathrm{yr}$ of model data only, while the spectra shown here are computed from the full 1500 yr. The white noise-like shape of the spectrum in the interannual range can be attributed to wind-forced Ekman currents contributing to the overturning cell. If the linear contribution of the winds to the overturning time series is removed (by means of regression analysis) the amount of high-frequency energy in the thermohaline circulation spectrum is lowered considerably. The resulting spectrum reveals a $\omega^{-4}$ frequency dependence being characteristic for a second-order stochastic process. There exists also a pronounced variability in the centennial range. These long-term modulations exhibit a damped behavior and can be attributed to long-term spinup effects, since the deep ocean is, although applying flux correction, not in perfect equilibrium. Meanwhile, the coupled experiment has been extended to more than 2000 yr. The deep ocean needs about 1400 yr to adjust. Afterward, both model drift and the centennial modulation disappear.

The mechanism leading to the interdecadal oscillation has been described in detail in Timmermann et al. (1998) and can be summarized as follows. Let us consider a situation $10 \mathrm{yr}$ before a maximum in the intensity of the meridional overturning cell occurs. During this time a well-developed negative SST anomaly covers almost the entire North Atlantic between $20^{\circ}$ and $50^{\circ} \mathrm{N}$. This pattern can be depicted from the first EOF of SST (Fig. 1c), the corresponding principal component leads the intensity of the THC by $10 \mathrm{yr}$. The spectrum of PC1 of SST shows also a marginally significant peak near 30 yr (Fig. 1d), but this spectrum is much more contaminated by high-frequency noise than that of the meridional overturning. Furthermore, a peak at about $15-y r$ period can be depicted, which is related to the gyre mode.

Although this mode is physically independent from the thermohaline driven one, it has some projection onto the EOF-pattern shown in Fig. 1c. However, the corresponding principal component shows coherence (with 10 year's lag) with the overturning index only at the interdecadal timescales. The atmosphere responds to these SST anomalies by a weakened North Atlantic Oscillation, as shown by the first EOF of Northern Hemisphere 500-hPa geopotential height (Fig. 1e). The occurrence of a spectral peak in the $500-\mathrm{hPa}$ geopotential height principal component at the relevant period (Fig. 1f) is a strong argument for the existence of a coupled air-sea mode. Furthermore, a NAO-like pattern was obtained also in atmosphere-only experiments with the ECHAM3 model forced by SST anomalies like those given in Fig. 1c. Although we believe that the mode has its origin in the North Atlantic, there are also clear atmospheric teleconnections to the North Pacific. The weakening of the NAO goes along with a strengthened Aleutian Low (Fig. 1e), and a coherent decadal signal is found also in Northern Pacific SST. The Pacific signal is standing in nature and strongly damped. It does not share many similarities with the Pacific decadal mode described by Latif and Barnett (1994, 1996), where propagating anomalies and ocean dynamics play a crucial role. Rather, our Pacific signal originates from atmospheric forcing by anomalous wind-driven Ekman currents, which can be related via atmospheric teleconnection to the North Atlantic. Although this atmospheric bridge is not understood up to now, it is an indication for an active role of the atmosphere in the North Atlantic at interdecadal timescales.

The weakened NAO feeds back onto the ocean. It is associated with negative freshwater flux anomalies (mainly stronger than normal evaporation) and oceanic Ekman salt transports that force positive salinity anomalies off Newfoundland and in the Greenland Sea. Oceanic convection is near normal at this time. The salinity anomalies amplify and propagate into the region of main oceanic convection, which is located south of Greenland. Here, they strengthen the convection in response to the enhancement in surface density $5 \mathrm{yr}$ later. At this time, SST and atmospheric anomaly patterns are not well developed and in a kind of transition state. The strengthened convection alters the density structure of the North Atlantic, which leads after an adjustment time of about $5 \mathrm{yr}$ to an enhancement of the THC. The subsequently increased poleward surface heat flux causes the formation of positive SST anomalies another $5 \mathrm{yr}$ later, which completes half a cycle.

The facts, that we find spectral peaks at the 30-yr period both in atmospheric and the oceanic quantities (Figs. 1b,d,f) and that these fluctuations are highly coherent (not shown) is indicative for the existence of a coupled air-sea mode. It gives rise for hope to make climate predictions on decadal timescales not only for the ocean but also for the atmosphere. However, the spectra reveal also significant differences in the amount of the high-frequency variability. While the overturning index exhibits relatively weak variability up to timescales of about a decade, the SST and especially the 500-hPa geopotential height anomalies are characterized by much more high-frequency variability. This has a strong impact on the predictability of these quantities as will be shown below.

Figures 2-4 show the results from the four forecast ensembles for the meridional overturning in the North Atlantic, the SST and the Northern Hemispheric 500$\mathrm{hPa}$ geopotential height. These figures are generated by projecting the individual ensemble members onto the EOFs presented in Fig. 1. Such a procedure has the advantage that irrelevant and unpredictable small-scale noise is filtered out. The panels on the left-hand side show the trajectories resulting from the projections of 

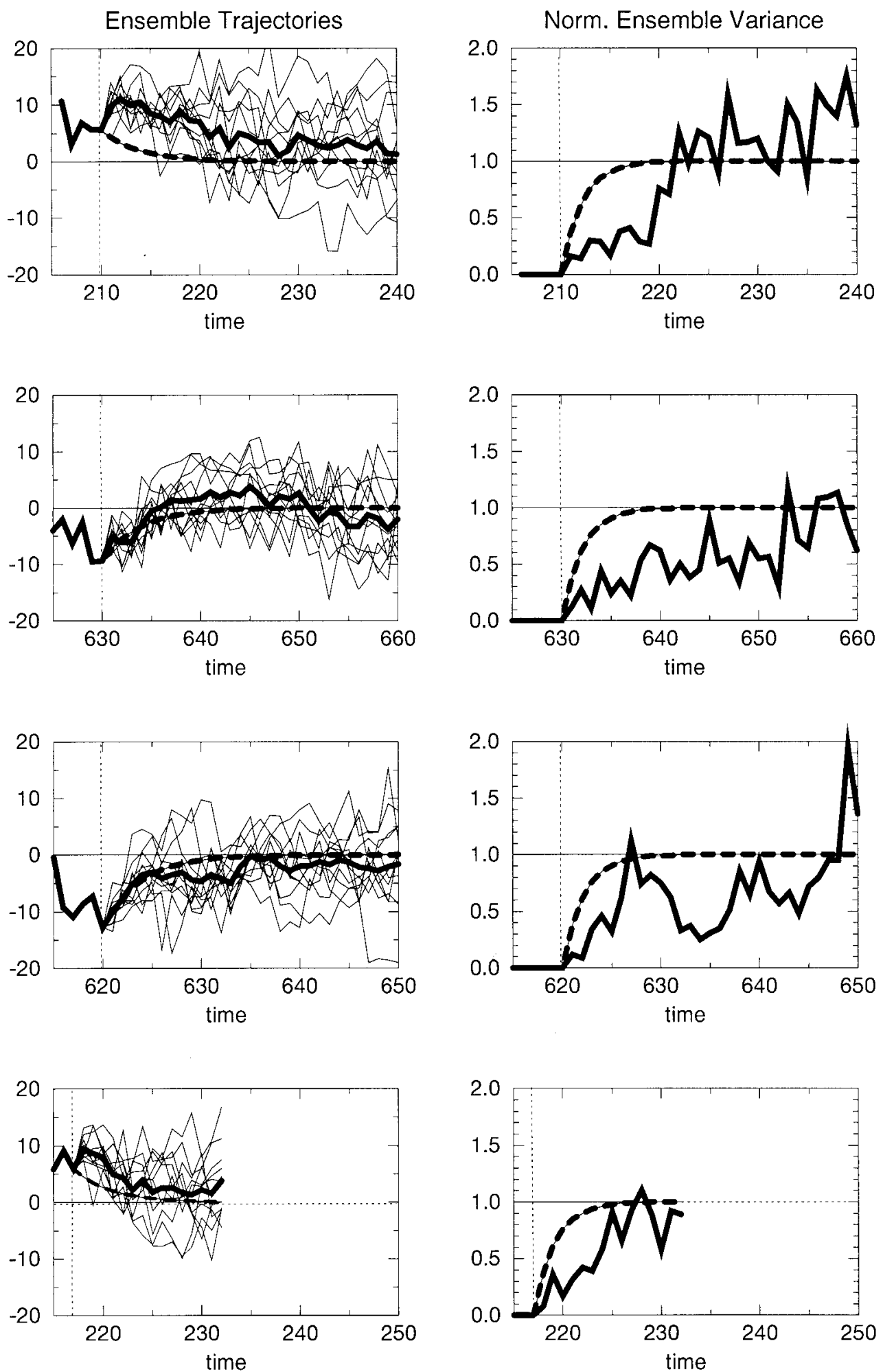

FIG. 2. Individual ensemble trajectories and ensemble mean (thick line) of projections onto the first EOF of the North Atlantic meridional overturning for four different forecast ensembles (left column) and time series of the respective normalized ensemble variances (right column). The thick dashed lines show the temporal behavior of fitted first-order autoregressive processes. 

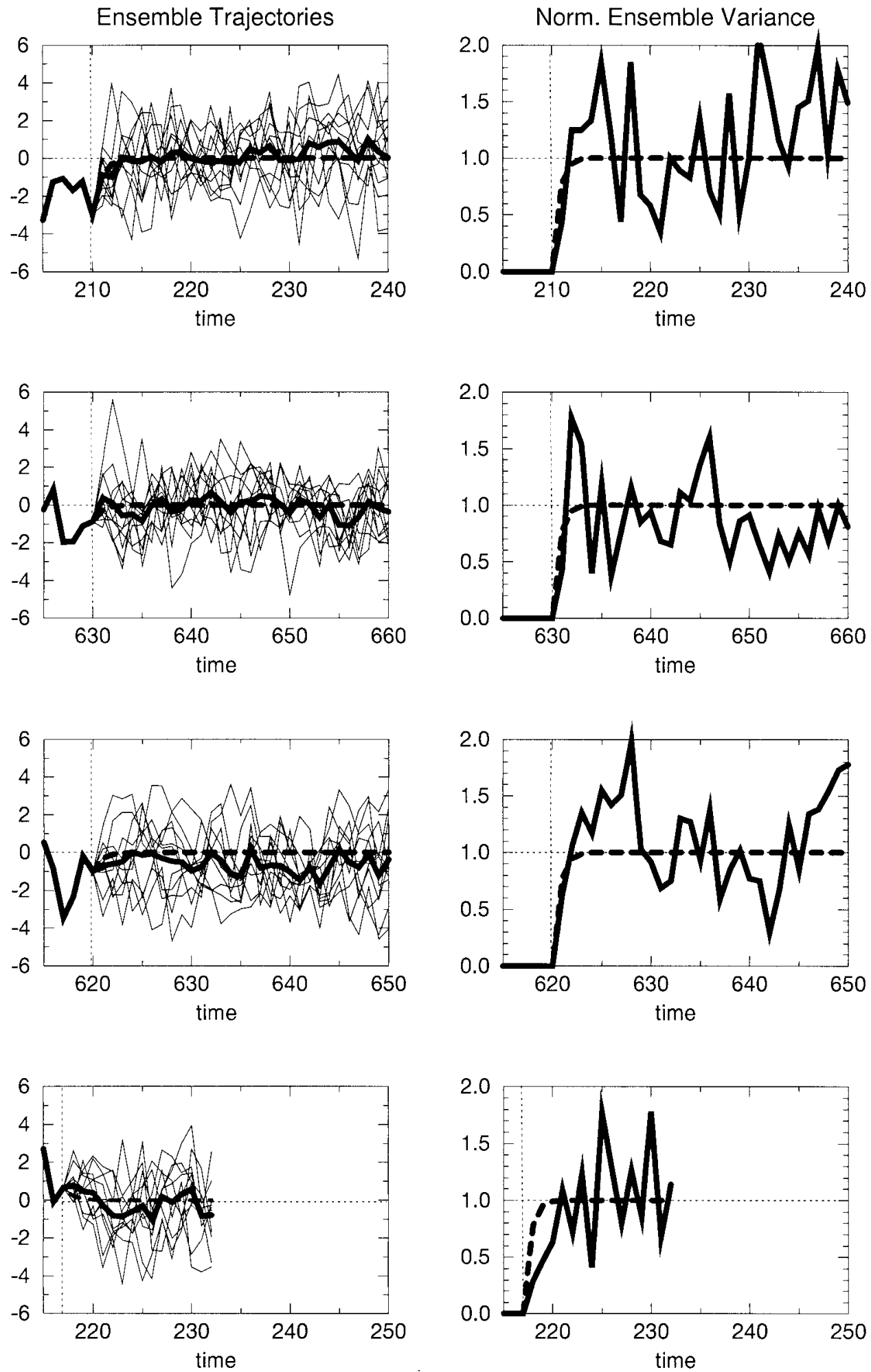

FIG. 3. Individual ensemble trajectories and ensemble mean (thick line) of projections onto the first EOF of Northern Atlantic SST anomalies for four different forecast ensembles (left column) and time series of the respective normalized ensemble variances (right column). The thick dashed lines show the temporal behavior of fitted first-order autoregressive processes. 

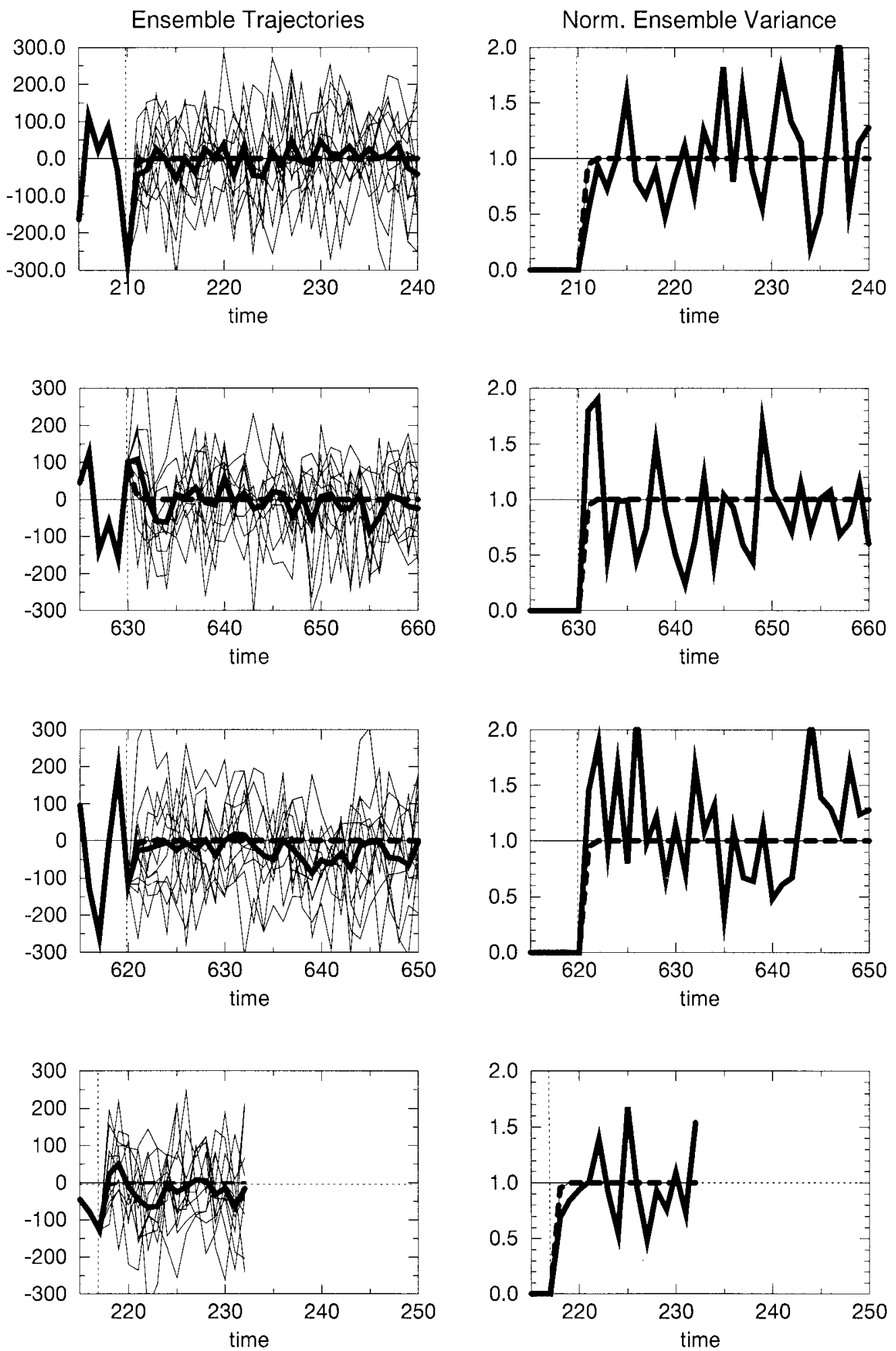

FIG. 4. Individual ensemble trajectories and ensemble mean (thick line) of projections onto the first EOF of Northern Hemispheric 500-hPa geopotential height SST anomalies for four different forecast ensembles (left column) and time series of the respective normalized ensemble variances (right column). The thick dashed lines show the temporal behavior of fitted first-order autoregressive processes. 
the individual ensemble members and the trajectory of the ensemble mean. The panels on the right display the spread within the ensembles as measured by the ensemble variance normalized by the climatological variance derived from the control run. When the normalized variance approaches unity, the variations between individual ensemble members are as large as typical variations in the control climate. The separation of ensemble variance and climatological variance can be used to define a predictability limit. However, the spread within a forecast ensemble that is tolerable is not an objective quantity. For an infinitely large forecast ensemble, the ensemble variance approaches the climatological variance asymptotically. Here we deal with only 11 realizations. This yields a rather crude estimate for the ensemble variance, and deviations from the climatological value might easily originate from sampling errors. For Gaussian variables the estimated variance obeys a $\chi^{2}$ distribution. For 11 realizations (10 degrees of freedom) the normalized ensemble variance has to remain below a value of $0.46(0.56)$ to be significantly different from climatology at the 95\% (90\%) significance level. Since these values are valid only for Gaussian variables, we have computed probability density distributions for the variance of random samples of the same size from the long-term control experiment. Deviations from an exact $\chi^{2}$ distribution generally were small. Thus, taking a value of about 0.5 for the normalized ensemble variance yields a relatively strict but statistically reliable predictability limit.

Since the stochastic climate model may serve as the null hypothesis for climate variability and predictability, we show also in Figs. 2-4 the results obtained by assuming equivalent first-order autoregressive processes. Analytical expressions for ensemble means and variances are given by exponential functions with damping coefficients estimated from the $\operatorname{lag}(1)$ autocorrelations derived from the control experiment. The estimate of the $\operatorname{lag}(1)$ autocorrelation has an inherent error associated with it. We have tested the statistical error bounds and found out that the deviations were generally very small. The $700 \mathrm{yr}$ of data are obviously enough to yield a reasonable estimate of the $\operatorname{lag}(1)$ autocorrelation.

Figure 2 displays the results for the meridional overturning in the North Atlantic. Ensembles 1 and 2 have been initialized from ocean states characterized by anomalously strong and weak overturning circulations. Ensemble 3 has been started also from a state with relatively weak circulation but within a phase with a rising tendency. Ensemble 4 is characterized by a relatively strong initial overturning circulation similar to ensemble 1 . However, ensemble 4 was not motivated by the overturning index. We have chosen this initial state, because it is characterized by an extremely strong Northern Atlantic SST anomaly. Ocean-atmosphere coupling should be strongest in such a phase and might have a positive effect on the predictive skill. The predictability of the meridional overturning is of the order of a decade.
The ensemble variance remains fairly small for about $10 \mathrm{yr}$ for ensemble 1, $20 \mathrm{yr}$ for ensemble 2, and slightly less than $10 \mathrm{yr}$ for the fourth ensemble. The predictability limit is not that clear for the third ensemble, since the ensemble variance increases rapidly after $7 \mathrm{yr}$ but drops again after $15 \mathrm{yr}$ and increases again after $20 \mathrm{yr}$. This behavior might be an expression of the small sample size. The temporal behavior of the ensemble variance clearly deviates from a pure red-noise process, which is characterized by $e$-folding times of about $2 \mathrm{yr}$. It is visible from the trajectory plots that the damping is much weaker than could be expected from a first order autoregressive [AR(1)] process. The ensemble means of the ensembles 1 and 4 even show an initial increase, while the ensemble mean of ensemble 2 exhibits an oscillatory behavior with the timescale of the interdecadal mode. In summary, the meridional overturning is highly predictable, even at decadal timescales.

Such a clear manifestation of the interdecadal mode in the predictability characteristics cannot be found in the other variables under consideration. Both the trajectories of the SST and 500-hPa geopotential height projections diverge very quickly. The normalized ensemble variances approach unity within a year and AR(1)-processes yield reasonable fits for these quantities. Only the fourth ensemble behaves slightly different. The SST seems to be predictable for about $3 \mathrm{yr}$ and the increase of the 500-hPa height variance is slower than in the other ensembles. However, its deviation from unity is not significant. The fourth ensemble was initialized from an extreme SST anomaly. This is not visible directly from the time series presented here. Crucial for the coupling, however, is the meridional SST gradient, to which EOF2 (not shown) contributes considerably. Although the predictability of surface quantities and atmospheric variables is generally very weak in the first three ensembles, the fourth ensemble gives at least an indication that the coupling between ocean and atmosphere may be relevant for decadal predictability in the North Atlantic climate system.

The results presented above are based on the leading EOFs computed from annual-mean data. We looked at the results of the predictability experiments in many different ways. The seasons have been investigated separately, since both the coupling strength between ocean and atmosphere and the internal noise level depend on the phase of the seasonal cycle. Long-term averages (5-yr running means) were considered, since such a filtering could enhance the signal-to-noise ratio of the seeked interdecadal signal. Moreover, different definitions of anomaly patterns have been investigated. Both the predictability of higher EOFs and of local indices were studied also. Time-dependent anomaly patterns defined by the local ensemble means were considered for prediction purposes as well. All these analyses confirmed the basic results presented above.

The four ensembles presented were all initialized in Northern Hemispheric winter. Another experiment was 


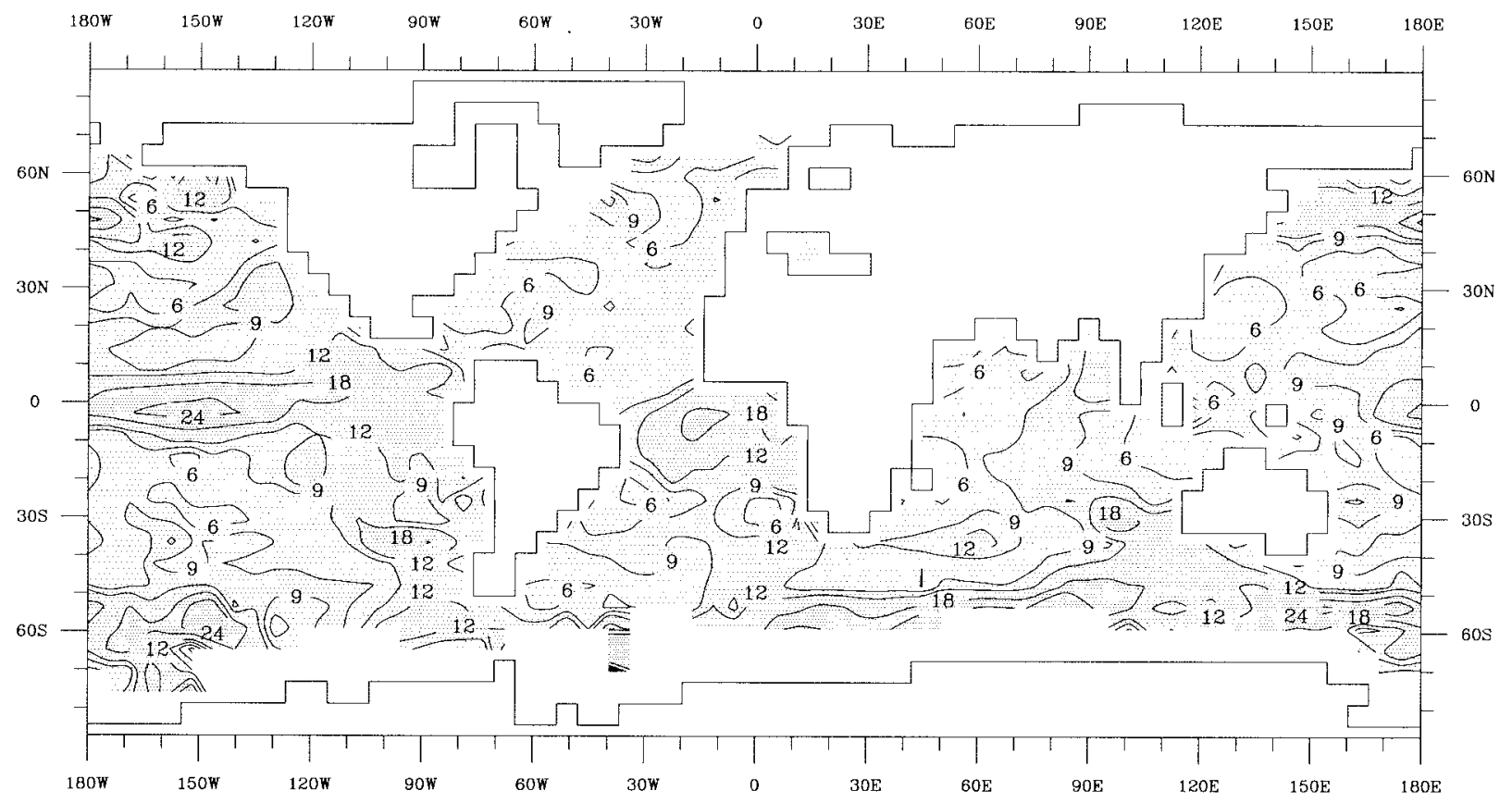

FIG. 5. Global distribution of exponential growth times (months) of the ensemble variance of SST as an average over four particular forecast ensembles.

started in summer to investigate whether the seasonality has any impact on the predictability limits. A seasonal dependence of the predictability on the initial season may be expected. The results of the summer experiment (not shown), however, were very similar to those of the winter experiments and yielded no hint for any seasonal dependence of predictability within the ECHAM3-LSG model.

Our predictability experiments highlight the ocean's role in generating long-term climate variability. This is reflected in the relatively long prediction timescales of the ocean circulation. Although the coupling between ocean and atmosphere seems also to play a key role in generating interdecadal variability, the coupling appears to be too weak (or equivalently the damping too strong) to influence the predictability of oceanic surface and atmospheric quantities at decadal timescales. The amount of internal generated high-frequency noise is too high and destroys the predictability at relatively long timescales. Therefore, the results of our predictability experiments can be viewed to be generally consistent with those shown by Griffies and Bryan (1997b). However, while the overturning exhibits comparable predictability in both models, the SST is more predictable in the GFDL model used by Griffies and Bryan (1997a,b). This points to a sensitivity of the predictability limits to the model formulation.

\section{Global predictability of interannual climate fluctuations}

The focus of the previous section was on the prediction of North Atlantic climate fluctuations, since the coupled model exhibits distinct decadal climate modes within this area, which may lead to enhanced predictability on decadal timescales. Since the predictability experiments were performed with a global coupled model, one could ask also whether there are other areas over the globe that exhibit some climate predictability deviating from that expected from a pure red noise process. To address this question the temporal evolution of the normalized ensemble variances has been investigated locally over the whole globe. Here we show results for global SSTs based on seasonal anomalies with respect to an annual cycle derived from the 700-yr control experiment. The spatio-temporal distribution of the ensemble variances for several prediction ensembles represents a huge amount of information that cannot be assessed so easily. Therefore, the temporal evolution of the ensemble variances is expressed in terms of a single parameter. Least-squares fits of exponential expressions as valid for first-order autoregressive processes have been applied to the time series of the normalized ensemble variances, and the exponential growth times were estimated. These can be directly compared with the decorrelation timescales estimated from the control experiment. Similar timescales obtained from these two different procedures should be viewed as a strong argument for the stochastic climate model null hypothesis in causing the model's SST variability. To further reduce the amount of information and to enhance statistical significance the resulting exponential growth times were averaged over all four ensembles.

Figure 5 displays the resulting global distribution of exponential growth times of the normalized ensemble 
variances for SST. Most areas over the globe yield growth times shorter than a year. These numbers are very similar to those obtained from the decorrelation timescales of the control experiments and consistent with the stochastic climate model scenario. The higher latitudes and the Tropics generally reveal a weaker damping than the subtropics. For example, in the northern North Atlantic and North Pacific and the tropical Atlantic we find exponential growth times of up to 12 months. These numbers are still consistent with the stochastic climate model scenario. The decorrelation timescales estimated from the control experiment show the same enhancement of the ocean's memory for these areas as the exponential growth times. The deepening of the ocean's mixed layer causes the enhanced thermal inertia in the higher latitudes. Positive wind feedbacks are the reason for the enhanced persistence in tropical SSTs. These results are in general agreement with those obtained by Manabe and Stouffer (1996), who computed $\operatorname{lag}(1)$ autocorrelations from a 1000-yr control integration with the GFDL coupled ocean-atmosphere model.

Only two areas clearly deviate in their statistics from the stochastic climate model scenario and exhibit exponential growth times much longer than expected from this simple picture. These areas are the tropical Pacific and the Southern Ocean in the region of the Antarctic Circumpolar Current (ACC). The numbers obtained for the Southern Ocean with values up to 24 months should be handled with caution. Although flux correction was applied, the model exhibits still some climate drift. This problem is worst in the Southern Ocean where a large amount of sea-ice is lost during the first $200 \mathrm{yr}$ of the control experiment (see Voss et al. 1998). This is associated with a damped centennial oscillation of the World Ocean circulation, which is also visible at the surface of the Southern Ocean. Such a centennial oscillation shows up on the timescale of the ensemble experiments as a strong trend, which all ensemble members have in common. We have tried to filter out this trend, but such a procedure is somewhat arbitrary, since the distinction between this trend and the signals to be predicted cannot be made objectively. Thus, we do not trust the results for the Southern Ocean.

The other region yielding relatively long predictability timescales is the equatorial Pacific. Averaged exponential growth times for SST of more than 24 months were simulated in the central equatorial Pacific. These are related to a variability mode, which shares many similarities with the ENSO phenomenon observed in the tropical Pacific (TLGV99). The model's tropical Pacific exhibits interannual oscillations with a characteristic period of about 5-8 yr. These oscillations can be understood within the framework of the delayed action oscillator theory (Suarez and Schopf 1988) and are related to equatorial wave dynamics and coupled airsea interactions. However, mixed layer processes and equatorial waves are only crudely represented within the ECHAM3-LSG model due the coarse resolution both in horizontal and vertical directions. Therefore, the variability level of the equatorial Pacific is underestimated by about a factor of 3 . However, the teleconnection patterns as they are known from observations are well reproduced by the coupled model.

Results of the predictability experiments for the model's upper ocean $(200 \mathrm{~m})$ heat content averaged over the NINO3 region $\left(150^{\circ}-90^{\circ} \mathrm{W}, 5^{\circ} \mathrm{S}-5^{\circ} \mathrm{N}\right)$ are given in Fig. 6 . Thermocline displacements related to the model ENSO are reflected in the upper-ocean heat content. Figure 6 shows like Figs. 2-4 the individual trajectories, ensemble means, and normalized variances for the four ensembles. Again, the respective curves for equivalent AR(1) processes are shown. Deviations from damped persistence are obvious for all four cases. For ensembles starting from an extreme phase of the model's ENSO (ensembles 1 and 3 ) the individual realizations show a clear tendency for a phase reversal. Ensemble 2 was started at an intermediate phase. All realizations within this ensemble show growing negative anomalies, which cannot be explained by a first-order model. After $1 \mathrm{yr}$ a La Niña state is attained and then all ensemble members show a common tendency for a phase reversal. The behavior of the fourth ensemble is not that clear. It was started from a small initial anomaly, and the anomalies of all realizations remain rather small for several years. The model's ENSO period of 5-8 yr is also visible within the time series of the ensemble means. The ensemble variances stay well separated from climatology [as measured by the $95 \%(90 \%)$ confidence limits of $0.46(0.56)]$ for 2-3 yr. These predictability properties are found also in atmospheric quantities. The model's Southern Oscillation index is also predictable for 2-3 yr highlighting the strong coupling between ocean and atmosphere and the low internal noise level of the tropical Pacific atmosphere. In summary, ENSO is predictable for a quarter to a third of the model's ENSO period, a result similar to that derived from more complex models (Latif et al. 1998).

\section{Discussion}

Ensemble forecast experiments have been performed with a global coupled GCM to investigate the predictability of the climate fluctuations on interannual to decadal timescales. The ensemble experiments reveal typical predictability timescales for the model ENSO of about 2-3 yr, that is, one-quarter to one-third of the oscillation period, both for NINO3 SST and for the atmospheric Southern Oscillation index. With respect to the model's North Atlantic Ocean the ensemble predictions yield also predictability timescales of one-quarter to one-third of the oscillation period of the 30-yr thermohaline mode, that is, of the order of a decade, for quantities describing the large-scale deep North Atlantic Ocean circulation. This behavior is not reflected in surface quantities and atmospheric variables. These cannot be distinguished in their predictability characteristics 

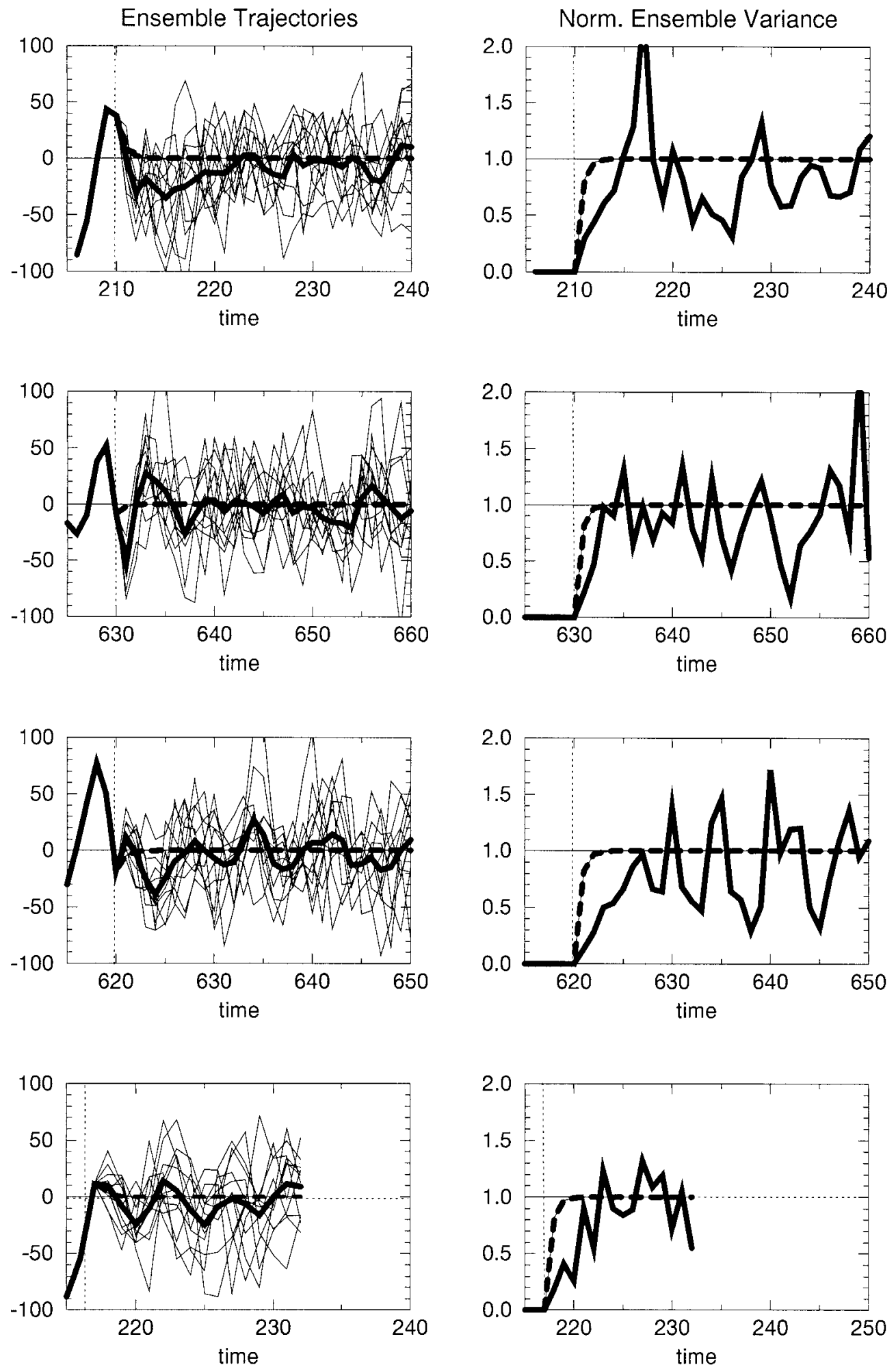

FIG. 6. Individual ensemble trajectories and ensemble mean (thick line) of NINO3 $\left(125^{\circ}-90^{\circ} \mathrm{W}, 5^{\circ} \mathrm{N}-5^{\circ} \mathrm{S}\right)$ upper-ocean heat content anomalies $(0-200 \mathrm{~m})$ for four different forecast ensembles (left column) and time series of the respective normalized ensemble variances (right column). The thick dashed lines show the temporal behavior of fitted first-order autoregressive processes. 


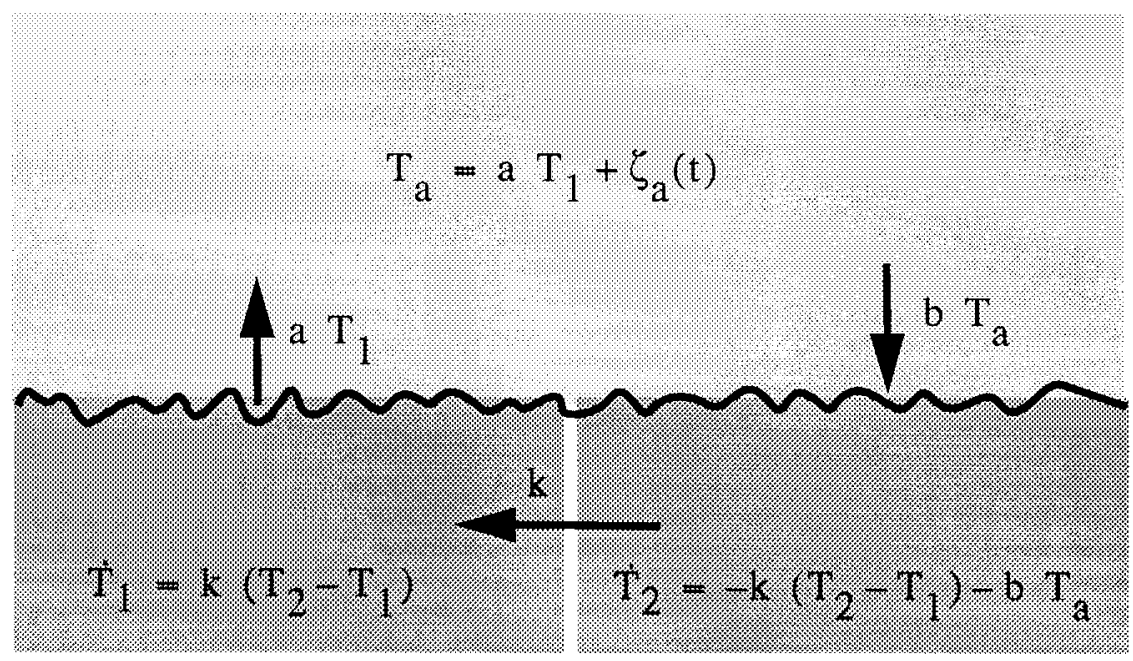

$$
\ddot{\mathrm{T}}_{1}+2 \mathrm{k} \dot{\mathrm{T}}_{1}+\mathrm{k} \text { a b } \mathrm{T}_{1}=-\mathrm{k} \mathrm{b} \zeta_{\mathrm{a}}(\mathrm{t})
$$

FIG. 7. A simple conceptual model for coupled ocean-atmosphere variability and predictability based on a second-order stochastic differential equation.

from those of a red noise process and yield typical predictability timescales of less than a year. This finding is true also for the rest of the world oceans. Global SST predictability, which is in the order of months to a year, can be sufficiently described by red noise. Most of the model's climate variability and predictability is consistent with Hasselmann's (1976) stochastic climate model concept.

However, the predictability results for the North Atlantic Ocean require a more detailed interpretation. The facts that the model exhibits a coupled ocean-atmosphere oscillation (Timmermann et al. 1998) and that this phenomenon is only predictable in the subsurface ocean but not at the surface or in the atmosphere are apparently in conflict with each other. The existence of a coupled mode means that there are simultaneous signals in both the ocean and the atmosphere. The atmosphere exhibits a specific response to certain SST anomalies. Since only the atmospheric initial conditions and not the SSTs were perturbed in the ensemble predictability experiments, a coupled feedback loop should also be visible in the predictability characteristics of atmospheric quantities. The predictability of the model ENSO clearly demonstrates such a behavior.

Two different views can be taken to explain the behavior of the North Atlantic Ocean. First, it might be possible that the two-way coupling between ocean and atmosphere is not really essential for the existence of the 30-yr oscillation. One could think of a stochastically forced ocean-only mode, where the atmosphere somehow reacts to the oceanic variations. However, this reaction must not necessarily feed back onto the ocean. The feedback to the ocean might be either nonexistent or weak and irrelevant. Within such a scenario, the coupling of ocean and atmosphere is not really relevant for the occurrence of a specific timescale. This view could explain also the limited predictability of atmospheric quantities. A slight increase only in predictability relative to the weather timescale can be expected due to the enhancement of atmospheric persistence. To test this hypothesis further, ocean-only experiments driven with temporally randomized atmospheric forcing taken from the coupled control integration, either in ensemble or control mode, would be necessary.

Second, also within the framework of a fully coupled ocean-atmosphere oscillator the coexistence of relatively long predictability of the deep ocean and almost vanishing predictability at the surface and in the atmosphere can be explained satisfactorily. It is the amount of high-frequency noise that crucially influences the predictability of the coupled system. The spectra shown in Fig. 1 exhibit different degrees of redness for the deep ocean, the ocean surface, and the atmosphere. The level of high-frequency noise relative to the quasiperiodic signal increases and destroys the predictability of the periodic signal at the surface and in the atmosphere.

The consequences on the predictability of the coupled system can be illustrated by means of a very simple conceptual model (Fig. 7). This model represents the simplest version of a coupled ocean-atmosphere oscillator. It is not our intention to derive a realistic model of how the dynamics of the THC might interact with the atmosphere in a coupled framework similar to that of Griffies and Tziperman (1995) for a stochastically driven ocean-only oscillator. Such an investigation, 
which would include both salinity and temperature effects influencing the meridional transports, is beyond the scope of this paper. We just want to illustrate the influence of noise on the variability and predictability of a coupled ocean-atmosphere mode.

The key elements for any coupled ocean-atmosphere oscillator are a kind of delaying process in the ocean representing the systems memory, a response of the atmosphere to the ocean, and a forcing of the ocean by the atmosphere to close the feedback loop. Since the atmosphere is conceptually not aligned with any memory, its forcing and response to the ocean have to be separated, either spatially or into different variables. Furthermore, atmospheric noise is required to overcome the inherent damping of the system. These elements can be found, for example, in the delayed oscillator concept for El Niño (Suarez and Schopf 1988), where propagating equatorial Rossby and Kelvin waves represent the systems memory, the atmospheric Walker cell is sensitive to eastern equatorial SST anomalies, and the aligned anomalous winds initiate those waves. In the case of our THC mode the atmosphere responds to specific SST anomalies, which in turn feeds back onto the ocean by generating salinity anomalies. The delayed response of the THC introduces the required memory into the system.

To keep things simple our conceptual model for a coupled ocean-atmosphere oscillator consists of two oceanic boxes and one atmospheric box characterized by state variables $T_{1}, T_{2}$, and $T_{A}$, for example, representing temperature anomalies. The memory of the system is given by the exchange between the two oceanic boxes. It is described by a diffusion term depending on the temperature difference and an exchange coefficient $k$. The atmospheric feedback loop is given by a response proportional to the ocean temperature $T_{1}$ by a coupling strength $a$ and a response of the ocean in box 2 proportional to the atmospheric temperature by a forcing parameter $b$. The atmospheric weather fluctuations are parameterized by white noise random forcing $\xi_{A}(t)$. To keep things simple the noise is thought to be independent from the atmospheric background state. Therefore, it can be handled as an additive term in the atmospheric temperature $T_{A}$ with the advantage to keep the system linear. In general, stochastic forcing should act also on box 1 . However, since this is not elementary to create coupled oscillations it is neglected here. The combination of these assumptions yields a second-order differential equation describing a damped linear harmonic oscillator under the influence of white noise forcing with a natural frequency $\omega_{0}^{2}=k b a$ and a damping coefficient $k$. Since a second-order differential equation is sufficiently determined by two parameters, $b$ can be set to unity without loss of generality and is kept only to maintain consistency in units. From this stochastic differential equation, expressions for autocovariance function $R(\tau)$, power spectra $S(\omega)$, and variances within hypo- thetical forecast ensembles $\sigma_{\mathrm{ENS}}^{2}(\tau)$ can be derived analytically. For the ocean temperature $T_{1}$ one obtains

$$
\begin{aligned}
R_{T_{1}}(\tau)= & \frac{b \sigma_{\zeta_{A}}^{2}}{4 a} e^{-k \tau}[\cos (\Omega \tau)+\sin (\Omega \tau)] \\
S_{T_{1}}(\omega)= & \frac{b^{2} k^{2} \sigma_{\zeta_{A}}^{2}}{4 k^{2} \omega^{2}+\left(\omega^{2}-\omega_{0}^{2}\right)^{2}} \\
\sigma_{\mathrm{ENS}_{T_{1}}^{2}}^{2}(\tau)= & \frac{b \sigma_{\zeta_{A}}^{2}}{4 a}\left\{1-\frac{e^{-2 k \tau}}{\Omega^{2}}\right. \\
& \left.\times\left[\omega_{0}^{2}-k^{2} \cos (2 \Omega \tau)+\Omega k \sin (2 \Omega \tau)\right]\right\},
\end{aligned}
$$

with $\sigma_{S_{A}}^{2}$ representing the variance of the white noise forcing and $\Omega=\left(\omega_{0}^{2}-k^{2}\right)^{1 / 2}$ the reduced frequency. The expressions for the autocovariance and ensemble variance consist of an exponential decay term and a harmonic contribution and are basically the same as those shown by Griffies and Bryan (1997b) for the case of an ocean-only oscillator. Since in climate applications the inherent damping is usually relatively strong, the exponential decay term is the dominant one. The spectrum is characterized by a $\omega^{-4}$ dependence and a spectral peak at the resonance frequency $\omega_{0}$. The respective quantities for the atmospheric temperature $T_{A}$ are given by

$$
\begin{aligned}
R_{T_{A}}(\tau) & =a^{2} R_{T_{1}}(\tau)+\sigma_{\zeta_{A}}^{2} \delta(\tau) \\
S_{T_{A}}(\omega) & =a^{2} S_{T_{1}}(\omega)+\sigma_{\zeta_{A}}^{2} \\
\sigma_{\mathrm{ENS}_{T_{A}}}^{2}(\tau) & =a^{2} \sigma_{\mathrm{ENS}_{T_{1}}^{2}}^{2}(\tau)+\sigma_{\zeta_{A}}^{2} \delta(\tau),
\end{aligned}
$$

where $\delta(\tau)$ denotes the Kronecker symbol with $\delta(0)=$ 1 and $\delta(\tau>0)=0$. Since the atmospheric temperature $T_{A}$ consists of a term proportional to $T_{1}$ and additive noise with vanishing autocorrelation, $R_{T_{A}}(\tau)$ and $\sigma_{\mathrm{ENS}_{T_{\Lambda}}}^{2}(\tau)$ exhibit a jump at $\tau=0$. The magnitudes of these jumps depend only on the absolute value of the coupling strength, since the strength of $T_{1}$ scales with the noise level. Figure 8 displays autocorrelation functions, spectra, and ensemble variances for values of the coupling strength $a$ of $0.2,1.0$, and 2.5, respectively. A value of $k=0.1 \mathrm{~K} \mathrm{yr}^{-1}$ yields oscillation periods in the decadal range. For easier comparison the results have been normalized using the oscillation period and climatological variance. The first case exhibits a highly damped behavior near the aperiodic limit. The second and the third cases show more or less pronounced oscillatory behavior depending on the ratio of damping coefficient $k$ to the resonance frequency $\omega_{0}$. The oceanic autocorrelation functions show a pronounced sign reversal, and the spectra exhibit clear spectral peaks. The oceanic ensemble variances grow relatively moderate and attain half of the climatological value within $0.2-$ 0.3 of the oscillation period depending on the damping of the system. This is totally different for the atmospheric temperature $T_{A}$. The ensemble variances jump 
oceanic temperature autocorrelation

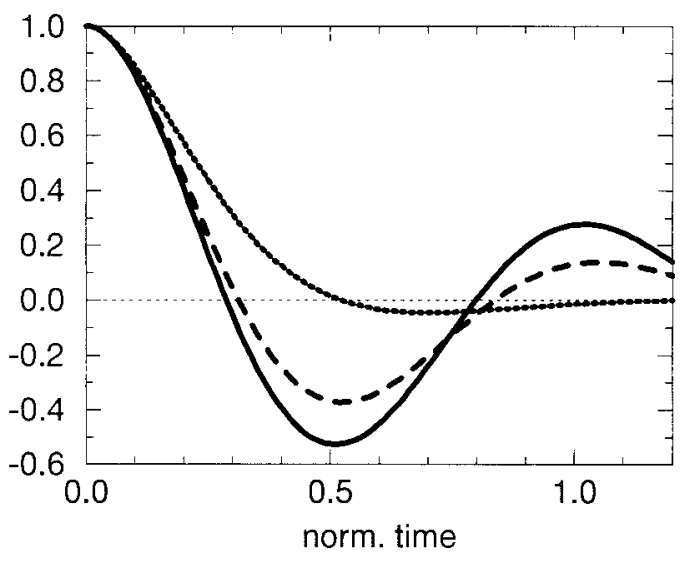

normalized spectra

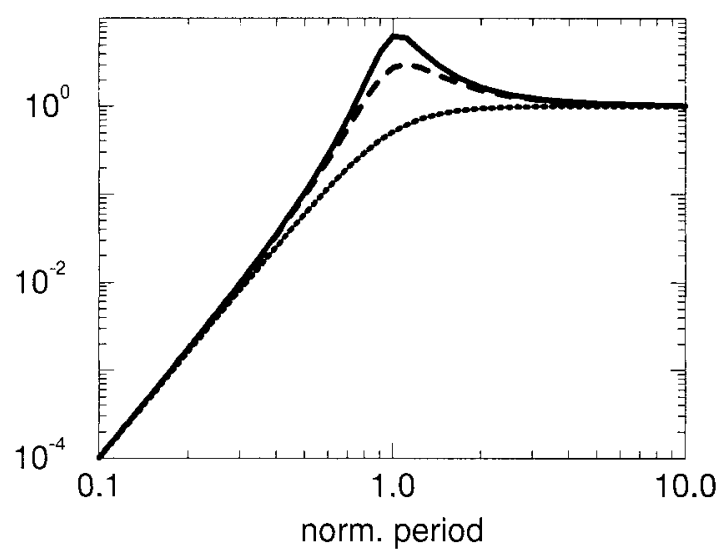

normalized ensemble variance

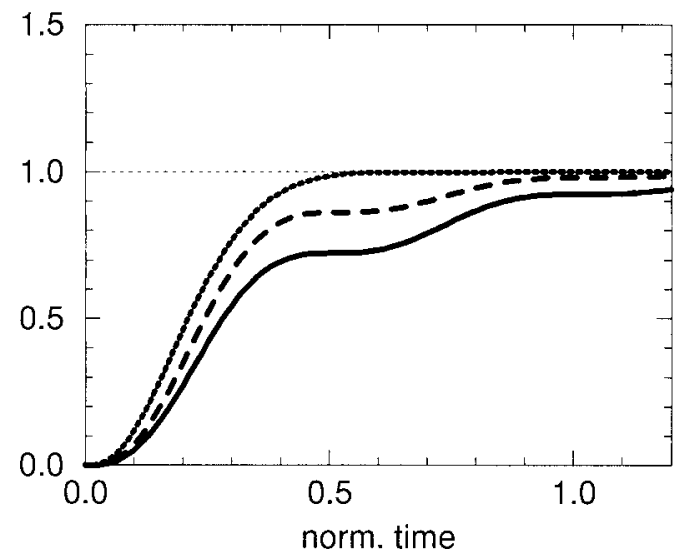

atmospheric temperature autocorrelation

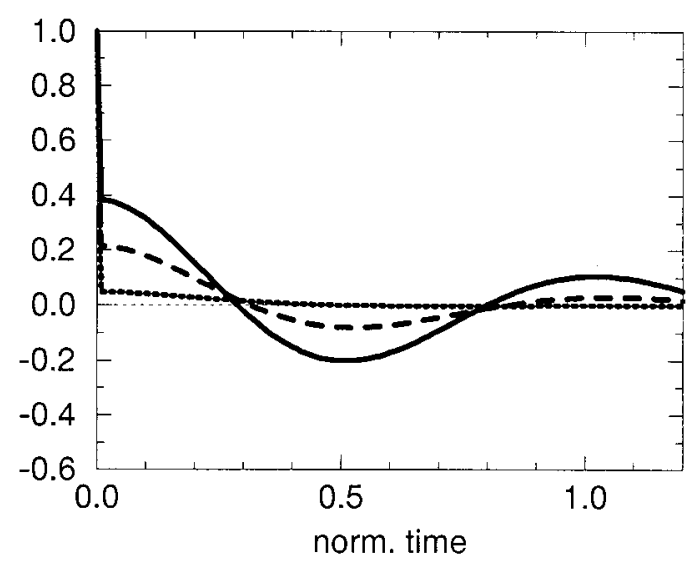

normalized spectra

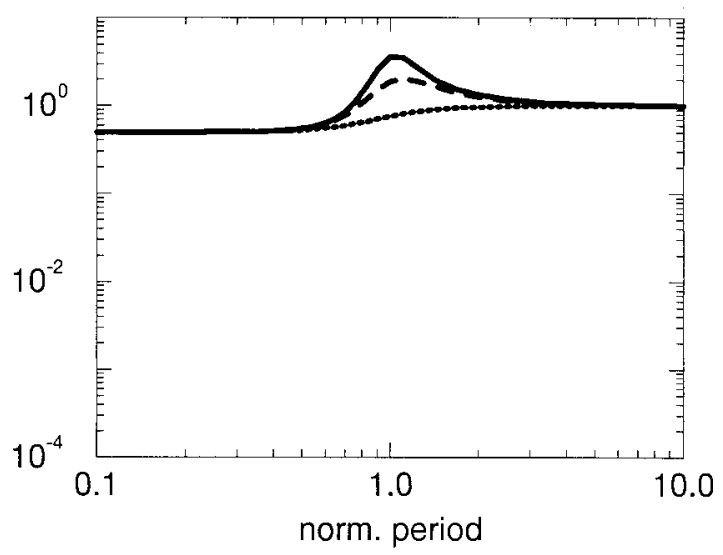

normalized ensemble variance

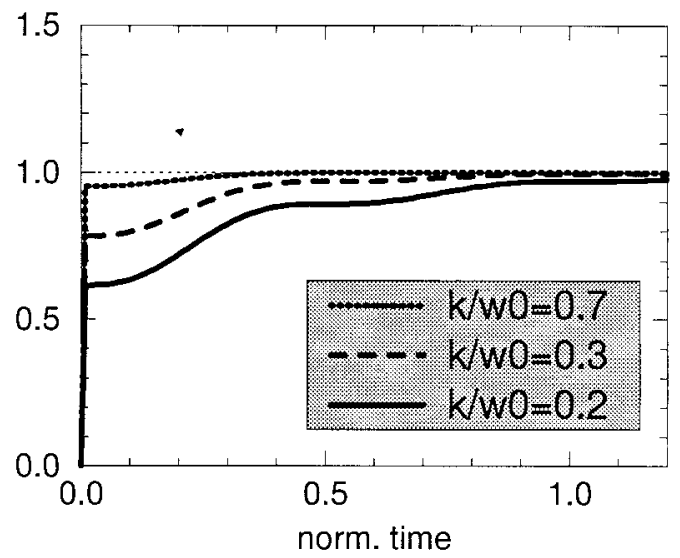

FIG. 8. Autocorrelation functions, normalized spectra, and ensemble variances for oceanic temperature $T_{1}$ and atmospheric temperature $T_{A}$ for coupling strengths $a$ of $0.2,1.0$, and 2.5 as derived from the analytical conceptual model. 
almost instantaneously (in the real atmosphere within the weather timescale) to a quite high value, which is almost indistinguishable from climatological variance. Only for very strong coupling the jump becomes weak enough to allow for predictability at longer lead times, as in the ENSO case described above. Although the jump due to the uncorrelated noise destroys almost any predictability, the spectra exhibit a similar shape as the ocean spectra in the low-frequency range. The additive white noise significantly affects the high-frequency range only, and the occurrence of the spectral peaks is hardly unchanged. Thus, our simple model is able to show that a coupled system might be able to oscillate, but that the presence of high-frequency weather noise can destroy almost any predictability of atmospheric quantities. Within such a coupled system the ocean acts as a very efficient low-pass filter highlighting the relevant decadal signal.

Our modeling results indicate that coupled oceanatmosphere oscillations can introduce a long-term memory into the extratropical climate system, but that the signal-to-noise ratio of the extratropical atmosphere is generally too low to gain relevant predictive skill from this memory. Optimal detection techniques (Venzke et al. 1999) could be used to enhance the signal-to-noise ratio within a given predictability ensemble and to detect a coupled signal against a noisy background. At this point it cannot be decided whether the model is able to reproduce the signal-to-noise ratios of the real climate system realistically since almost nothing is known about this quantity from observations. From the present knowledge it cannot be decided if the observed decadal and interdecadal climate fluctuations (e.g., Deser and Blackmon 1993; Kushnir 1994) are just occurring randomly or if they are an expression of any regularity. However, it is very likely that deficiencies of our coupled model might lead to an imperfect reproduction of the real world. The crude mixed-layer representation of the ECHAM3-LSG model might cause an underestimation of decadal surface temperature signals in the ocean. Furthermore, the rather coarse T21 resolution might cause both a misrepresentation of internal atmospheric variability and atmospheric response to SST anomalies. Similar experiments with more sophisticated models will give more insight into the predictability of extratropical climate fluctuations.

Acknowledgments. We would like to thank Dr. S. M. Griffies and Dr. U. Mikolajewicz for making valuable comments and suggestions on our manuscript. This work was sponsored by the Bundesminister für Forschung und Technologie. Further support was provided by the European Union's PROVOST and SINTEX projects. The extended control integration with the ECHAM3-LSG model has been performed by the staff of the Deutsches Klimarechenzentrum.

\section{REFERENCES}

Barnett, T. P., and R. Preisendorfer, 1987: Origins and levels of monthly forecast skill for the United States surface air temperatures determined by canonical correlation analysis. Mon. Wea. Rev., 115, 756-773.

Barnston, A. G., 1994: Linear statistical short-term climate predictive skill in the Northern Hemisphere. J. Climate, 7, 1513-1564.

Chang, P., L. Ji, and H. Li, 1997: A decadal climate variation in the tropical Atlantic Ocean from thermodynamic air-sea interactions. Nature, 385, 516-518.

Cubasch, U., G. C. Hegerl, R. Voss, J. Waskewitz, and T. J. Crowly, 1997: Simulation of the influence of solar radiation variations on the global climate with an ocean-atmosphere general circulation model. Climate Dyn., 13, 757-767.

Delworth, T., S. Manabe, and R. Stouffer, 1993: Interdecadal variability of the thermohaline circulation in a coupled ocean-atmosphere model. J. Climate, 6, 1993-2011.

$\longrightarrow, \ldots$, and 1997: Multidecadal climate variability in the Greenland Sea and surrounding regions: A coupled model simulation. Geophys. Res. Lett., 24, 257-260.

Deser, C., and M. L. Blackmon, 1993: Surface climate variations over the North Atlantic Ocean during winter: 1900-1989. J. Climate, 6, 1743-1753.

Frankignoul, C., P. Müller, and E. Zorita, 1997: A simple model of the decadal response of the ocean to stochastic wind forcing. $J$. Phys. Oceanogr., 27, 1533-1546.

Griffies, S. M., and E. Tziperman, 1995: A linear thermohaline oscillator driven by stochastic atmospheric forcing. J. Climate, 8, 2440-2453.

_ , and K. Bryan, 1997a: Ensemble predictability of simulated North Atlantic inter-decadal variability. Science, 275, 181-184.

— , and — 1997b: A predictability of simulated North Atlantic multidecadal variability. Climate Dyn., 13, 459-487.

Grötzner, A., M. Latif, and T. P. Barnett, 1998: A decadal climate cycle in the North Atlantic Ocean as simulated by the ECHO coupled GCM. J. Climate, 11, 831-847.

Gu, D., and S. G. H. Philander, 1997: Interdecadal climate fluctuations that depend on exchanges between the Tropics and extratropics. Science, 275, 805-807.

Hasselmann, K., 1976: Stochastic climate models. Part I: Theory. Tellus, 28, 473-485.

Hegerl, G. C., K. Hasselmann, U. Cubasch, J. F. B. Mitchell, E. Roeckner, R. Voss. and J. Waszkewitz, 1997: Multi-fingerprint detection and attribution analysis of greenhouse gas, gas-plusaerosol and solar forced climate change. Climate Dyn., 13, 613634.

Kushnir, Y., 1994: Interdecadal variations in the North Atlantic sea surface temperature and associated atmospheric conditions. $J$. Climate, 7, 141-157.

Latif, M., and T. P. Barnett, 1994: Causes of decadal variability over the North Pacific and North America. Science, 266, 634-637.

- and - 1996: Decadal variability over the North Pacific and North America: Dynamics and predictability. J. Climate, 9, $2407-2423$

— - and Coauthors, 1998: A review of the predictability and prediction of ENSO. J. Geophys. Res., 103, 14 375-14 393

Lorenz, E. N., 1963: Deterministic nonperiodic flow. J. Atmos. Sci., 20, 130-141.

Maier-Reimer, E., and U. Mikolajewicz, and K. Hasselmann, 1993: Mean circulation of the Hamburg LSG model and its sensitivity to the thermohaline surface forcing. J. Phys. Oceanogr., 23, 731757.

Manabe, S., and R. J. Stouffer, 1996: Low-frequency variability of surface air temperature in a 1000-yr integration of a coupled ocean-atmosphere model. J. Climate, 9, 376-393.

Mikolajewicz, U., and E. Maier-Reimer, 1990: Internal secular variability in an OGCM. Climate Dyn., 4, 145-156.

Roeckner, E. K., and Coauthors, 1992: Simulation of the present-day climate with the ECHAM model: Impact of model physics and 
resolution. Rep. 93, 171 pp. [Available from Max-Planck-Institut für Meteorologie, Bundestr. 55, D-20146 Hamburg, Germany.]

Sausen, R., K. H. Barthels, and K. Hasselmann, 1988: Coupled oceanatmosphere models with flux correction. Climate Dyn., 2, 154-163.

Schiller, A., U. Mikolajewicz, and R. Voss, 1997: The stability of the thermohaline circulation in a coupled ocean-atmosphere general circulation model. Climate Dyn., 13, 325-347.

Suarez, M. J., and P. S. Schopf, 1988: A delayed action oscillator for ENSO. J. Atmos. Sci., 45, 3283-3287.

Timmermann, A., 1999: Modes of variability as simulated by a global climate model. Dissertation 64, 92 pp. [Available from Max-
Planck-Institut für Meteorologie, Bundestrasse 55, D-20146 Hamburg, Germany.]

— M. Latif, A. Grötzner, and R. Voss, 1998: Northern Hemispheric interdecadal variability: A coupled air-sea mode. J. Climate, 11, 1906-1931.

Venzke, S., M. R. Allen, R. T. Sutton, and D. P. Rowell, 1999: The atmospheric response over the North Atlantic to decadal changes in sea surface temperature. J. Climate, 12, 2562-2584.

Voss, R., R. Sausen, and U. Cubasch, 1998: Periodically synchronously coupled integrations with the atmosphere-ocean general circulation model ECHAM3/LSG. Climate Dyn., 14, 249-266. 
Copyright of Journal of Climate is the property of American Meteorological Society and its content may not be copied or emailed to multiple sites or posted to a listserv without the copyright holder's express written permission. However, users may print, download, or email articles for individual use. 